CLINICAL STUDY

\title{
Characterization of the humoral immune response to islet antigen 2 in children with newly diagnosed type 1 diabetes
}

\author{
Anna Mäkinen ${ }^{1}$, Taina Härkönen ${ }^{1}$, Jorma Ilonen ${ }^{2,3}$, Mikael Knip ${ }^{1,4}$ and the Finnish Pediatric Diabetes Register \\ ${ }^{1}$ Scientific Laboratory, Hospital for Children and Adolescents, University of Helsinki, Helsinki, Biomedicum I, PO Box 63, FI-O0014 Helsinki, Finland, \\ ${ }^{2}$ Immunogenetics Laboratory, University of Turku, Turku, Finland, ${ }^{3}$ Department of Clinical Microbiology, University of Kuopio, Kuopio, Finland and \\ ${ }^{4}$ Department of Pediatrics, Tampere University Hospital, Tampere, Finland \\ (Correspondence should be addressed to M Knip who is now at Hospital for Children and Adolescents, University of Helsinki, PO Box 281, FI-O0O29 \\ HUCH, Helsinki, Finland; Email: mikael.knip@hus.fi)
}

\begin{abstract}
Objective: To characterize the humoral immune response to islet antigen 2 (IA-2) in patients with newly diagnosed type 1 diabetes (T1D), we compared the profile of epitope- and isotype-specific IA-2 antibodies (IA-2A) between children with a humoral immune response restricted to IA-2 and children with a broad response including insulin autoantibodies (IAA) and antibodies to glutamic acid decarboxylase (GADA) in addition to IA-2A.

Methods: The study subjects $(n=100)$ were derived from a consecutive series of 1108 patients from the Finnish Pediatric Diabetes Register (investigators listed in the Appendix). Islet cell antibodies, IAA, GADA, total IA-2A levels, IA-2/IA-2 $\beta$ epitopes, and isotypes were measured, and human leukocyte antigen (HLA) genotypes were analyzed.

Results: There were no significant differences between the two groups in the frequency or levels of epitope-specific IA-2A. Those with an IA-2-restrictive response tested positive more frequently for IgAIA-2A $(P=0.001)$, had higher titers of IgE-IA-2A $(P=0.025)$, tested positive for more IA-2A isotypes than the broad responders $(P=0.04)$, and carried the high-risk $H L A-(D R 4)-D Q B 1^{*} 0302$ haplotype more frequently than those with a broad antibody response $(P=0.019)$.

Conclusions: These data show that children with newly diagnosed T1D, who test positive only for IA-2A out of the three molecular antibodies predictive of T1D, have a broader IA-2-specific isotype response and stronger association with the high-risk HLA haplotype than those testing positive for all three molecular antibodies. This may be indicative of a different pathogenetic mechanism in those with their humoral immune response restricted to IA-2 at the time of diagnosis.
\end{abstract}

European Journal of Endocrinology 159 19-26

\section{Introduction}

Type 1 diabetes (T1D) is a chronic autoimmune disease characterized by an asymptomatic prediabetic period, which may last for years (1). During this period, autoantibodies to $\beta$-cells located in the pancreatic islets can be detected from the peripheral circulation as a marker of an ongoing T-cell-mediated destructive process in the islets. Such autoantibodies can be used for the identification of individuals at high risk for developing T1D (1). A series of autoantigens present in the $\beta$-cells have been characterized, the major ones comprising insulin, glutamic acid decarboxylase (GAD), and the protein tyrosine phosphatase (PTP)-like proteins, islet antigen 2 (IA-2), and $2 \beta$ (IA-2 $\beta$ ). Both IA-2 antigens are enzymatically inactive transmembrane proteins and they are assumed to play a role in the regulation of insulin secretion $(2,3)$. Humoral autoimmunity is directed toward the intracellular portion of these molecules. Several autoantigenic domains have been identified, including the juxtamembrane region (JM) of the IA-2 protein (amino acids (aa) 601-682), the PTP domain of the IA-2 protein (aa 687-979), and the PTP domain of the IA-2 $\beta$ protein (aa 741-1033). Autoantibodies that cross-react between the IA- 2 and IA- $2 \beta$ PTP domains (crPTP) are also common (4-8). Antibodies to the IA-2 $\beta$-specific epitopes are less frequent than those specific for the IA-2 epitopes, and have therefore been implicated to arise by epitope spreading secondary to the IA-2-specific humoral response (5, 7-9).

It has been suggested that the autoimmune reaction toward islet cells may start as a benign T-helper 2 (Th2)biased response that later polarizes into a destructive Th1 response leading to overt T1D (10). The analysis of isotype-specific autoantibodies to a given antigen may potentially provide the means of assessing the polarization of the autoantigen-specific immune response.

Multiple chromosomal loci have been associated with either predisposition to or protection from T1D. 
The most important genes are located on the short arm of chromosome 6 in the HLA region. It has been estimated that about $50 \%$ of the familial clustering of T1D can be explained by these genes (11). IA-2 antibodies (IA-2A) have been associated with one of the strongest haplotypes predisposing to T1D, namely the (DR4)-DQB1*0302 haplotype $(11,12)$.

In this study, we set out to further characterize the humoral immune response to the IA-2 antigen in children with newly diagnosed T1D with different islet autoantibody profiles, i.e. patients with their response restricted to IA-2 and those with additional responses to both of the other two biochemically defined major autoantigens. We hypothesized that those with a broad general autoantibody response would also have a broader IA-2-specific response than those with their response restricted to IA-2.

\section{Subjects and methods}

\section{Subjects}

The study subjects were derived from the Finnish Pediatric Diabetes Register and Biobank (investigators listed in the Appendix) that comprise data and samples from children and adolescents with newly diagnosed T1D and from their first-degree relatives. The register covers at least $82 \%$ of all children and adolescents under the age of 16 years diagnosed with T1D during the time period from June 1, 2002 to September 30, 2005 in Finland based on the use of the register of the National Social Insurance Institute as a secondary source. Blood samples for the isolation of serum, cells, and DNA are drawn for the Biobank as soon as possible after the diagnosis. The register includes information on the family history of T1D and data on the degree of metabolic decompensation at the time of diagnosis. Islet cell antibodies (ICA), insulin autoantibodies (IAA), autoantibodies to GAD (GADA), and IA-2A are measured from the serum samples of the diabetic children and their family members and their HLAgenotypes are analyzed. The parents of the children as well as each child 10 years of age or older gave their informed written consent. The protocol has been approved by the Ethical Committee of the Hospital District of Helsinki and Uusimaa.

By April 2005 the Register and Biobank comprised data and samples from 1108 children with T1D diagnosed under the age of 16 years and their firstdegree relatives. A majority of these subjects were males $(638 ; 57.6 \%)$, and the mean age at diagnosis was 8.30 years with a median of 8.40 years (range 0.01-15.99). Almost $75 \%$ of the children and adolescents (825; $74.5 \%)$ tested positive for IA-2A with a median level of 96.6 relative units (RU) (range 0.88-553.3), 719 (64.9\%) had GADA (median 41.4 RU, range 5.41812.4), and $528(47.7 \%)$ had IAA (median 11.7 RU, range 2.86-2379.4). Out of the 1108 children, 149 (13.4\%) had IA-2A as their only detectable biochemically characterized autoantibody, and 297 (26.8\%) tested positive for all three molecular autoantibodies (IA-2A, IAA, and GADA). Isolated IAA-positivity $(38 / 1108,3.4 \%)$ and GADA positivity $(80 / 1108$, $7.2 \%)$ were less frequent $(P<0.001)$ than isolated IA$2 \mathrm{~A}$ positivity. In the present study, we included 50 children with newly diagnosed T1D who tested positive for IA-2A but negative for the other two molecular autoantibodies, i.e. IAA and GADA, and who could be matched with a child with newly diagnosed diabetes with all three molecular autoantibodies. These IA-2restrictive responders and broad responders were matched for sex, age $( \pm 1$ year), and IA-2A titer $( \pm$ 20 RU). A majority of the 100 subjects were males $(n=64)$. The age of the children ranged from 1.45 to 15.95 years with a median of 9.52 years and a mean of 9.71 years (3.01; s.D.). The samples were analyzed for total IA-2A levels, IA-2/IA- $2 \beta$ epitopes (JM, PTP, $\beta$ PTP, crPTP), IgG subclasses (IgG1, IgG2, IgG3, and IgG4), and other isotypes (IgA, $\operatorname{IgE}$, and $\operatorname{IgM})$.

\section{Methods}

Antibodies IA-2A were analyzed with a RIA as described previously (13). Antibody levels were expressed as RU based on a standard curve derived from a pool of samples strongly positive for IA-2A and diluted in normal human serum (NHS). The disease sensitivity of this assay was $72 \%$ and disease specificity $100 \%$ according to the 2005 Diabetes Autoantibody Standardization Program (DASP). All samples initially tested as having IA-2A levels above $90 \mathrm{RU}$ were diluted in NHS to obtain reliable results from the linear part of the standard curve. IAA and GADA were analyzed with specific radiobinding assays $(14,15)$ with disease sensitivities of 44 and $82 \%$ and specificities of 98 and $97 \%$ respectively according to DASP 2005 . ICA were quantified by a standard indirect immunofluorescence method (16). More than 350 non-diabetic Finnish children and adolescents were tested for IAA, GADA, and IA-2A to determine the limits of positivity defined as the 99th percentiles, and being 2.80, 5.36, and 0.77 RU, respectively.

\section{Epitope- and isotype-specific IA-2/IA-2 $\beta$ antibodies} Epitope- and isotype-specific antibodies to IA- 2 and IA- $2 \beta$ proteins were analyzed as described in detail previously (17). The protocol for analyzing epitope-specific antibodies was essentially identical to that used for IA-2A except that epitope-specific radioligands IA-2 PTP $_{687-979}$, IA-2 $\beta$ PTP $_{741-1033}$, IA-2 389-779, and IA- $2_{601-682} / \mathrm{IA}-2 \beta_{737-1033}$ were used. To identify crossreactive antibodies and antibodies specific to a single epitope the sera were preincubated with unlabeled 
IA-2 and IA-2 $\beta$ proteins expressed in Escherichia coli. After direct binding and competition experiments, epitopespecific antibodies were classified into four reactivities, namely IA-2 JM, specific IA-2 PTP, specific IA-2 $\beta$ PTP, and cr IA-2 PTP/IA-2 $\beta$ PTP antibodies. Isotype- and subclassspecific antibodies were analyzed by using precipitation with biotinylated isotype and subclass-specific monoclonal antibodies linked to streptavidin agarose. Isotypespecific antibody results were expressed as SDS as defined previously (17), and a level of 3 SDS or more was considered positive.

HLA typing HLA typing data were available for 95 out of 100 subjects. Typing was performed with a technique based on solution hybridization with lanthanide-labeled oligonucleotide probes using time-resolved fluorometry for detection (18). A low-resolution full-house genotyping for major $D R-D Q$ haplotypes covering $D R 4$ subtypes was also performed as described earlier (18). According to the HLA typing results, the subjects were classified into five risk categories depending on their genetic risk of developing T1D. The highest risk genotype (risk group 4) included subjects carrying two different riskassociated haplotypes ((DR3)-DQA ${ }^{*} 05-D Q B 1^{*} 02$ and $\left.D R B 1{ }^{*} 0401 / 2 / 4 / 5-D Q A 1 * 03-D Q B 1 * 0302\right)$, risk group 3 , those who were homozygous for a risk-associated haplotype, had DRB1*0401/2/4/5-DQA1*03-DQB1 *0302 combined with a neutral haplotype or had the (DR3)-DQA1*05-DQB1*02/(DR9)-DQA1*03-DQB1 *0303 genotype. Risk group 2 comprised other genotypes combining (DR3)-DQA $1^{*} 05-D Q B 1 * 02$ with a neutral haplotype and the $D R B 1^{*} 0401 / 2 / 4 / 5$ DQA $1^{*} 03-D Q B 1 * 0302 /(D R 13)-D Q B 1 * 0603$ genotype. Risk group 1 covered other genotypes where a risk haplotype was combined with a protective one ((DR15)DQB1*0602, (DR11/12/13)-DQA1*05-DQB1*0301, (DR 14)-DQB1*0503, (DR7)-DQA1*0201-DQB1*03 $03)$ as well as combinations of two neutral haplotypes. Risk group 0 included the subjects who had a combination of protective haplotypes or a protective haplotype associated with a neutral one. The classification is based on a study of 622 Finnish nuclear families with a child affected by T1D (18). A dichotomous classification was also applied, according to which risk groups 0-2 comprised a category 'at low risk of developing T1D,' while risk groups 3 and 4 included those at 'high risk of developing T1D.'

Markers of metabolic decompensation at diagnosis The markers of the degree of metabolic decompensation at diagnosis were measured in the local laboratories. These parameters included $\mathrm{pH}$, blood glucose, and $\beta$-hydroxybutyrate levels. Data on blood glucose concentrations were missing in two subjects and those on blood $\mathrm{pH}$ in four subjects. Serum $\beta$-hydroxybutyrate concentrations were missing in 13 subjects.

Data handling and statistical analysis When evaluating the frequencies of different epitope- and isotype-specific IA-2A and HLA markers between groups, we used cross-tabulation and $\chi^{2}$-statistics with Yates continuity correction when appropriate. MannWhitney U-test was used to compare different variables between groups. The differences in the antibody levels within groups were evaluated with Wilcoxon's rank-sum test. A two-tailed $P$ value of 0.05 or less was considered statistically significant. All the statistical analyses were performed with the 12.0.1 version of the SPSS statistical software package (SPSS Inc., Chicago, IL, USA).

\section{Results}

\section{Autoantibodies}

As defined by our inclusion criteria, all the children included in this study were positive for IA-2A, and broad responders tested in addition positive for IAA and GADA. The median IA-2A level was 92.6 RU (range 60.5-149.1 RU) among the IA-2-restrictive responders and 96.6 RU (range 49.8-153.4) among the IA-2broad responders $(P=0.55)$ based on the Biobank measurements. The median GADA titer was 35.3 RU (range 8.3-216.5) and the median IAA level was 8.0 RU (range 3.39-1057.2 RU) among the broad responders. There was no difference in the frequency of ICA between the two groups; only three children among the IA-2-restrictive responders and one among the broad responders tested negative for ICA $(P=0.61)$. However, the ICA titers were higher in the group of broad responders than in those with an IA-2-restrictive response (medians 44 vs 12 Juvenile Diabetes Foundation (JDF) units, $P<0.001$ ).

We reanalyzed total IA-2A levels after diluting all the samples initially measured to have an IA-2A titer of 90 $\mathrm{RU}$ or more. As a result, these antibody levels were higher than those previously seen in the analyses for the Biobank dataset (medians 161.1 vs 96.2 RU, $P<0.001)$. There was no difference in the total IA-2A titers between the restrictive and broad responders. The median IA-2A level was 171.1 RU (range 37.9-4523 RU) in the restrictive responders and 140.6 RU (range 36.2-8241 RU) in the broad responders.

\section{Epitope-specific antibodies}

There were no differences in the epitope-specific responses between the IA-2-restrictive and -broad responders. The two groups did not differ in terms of epitope-specific antibody frequencies or antibody levels 


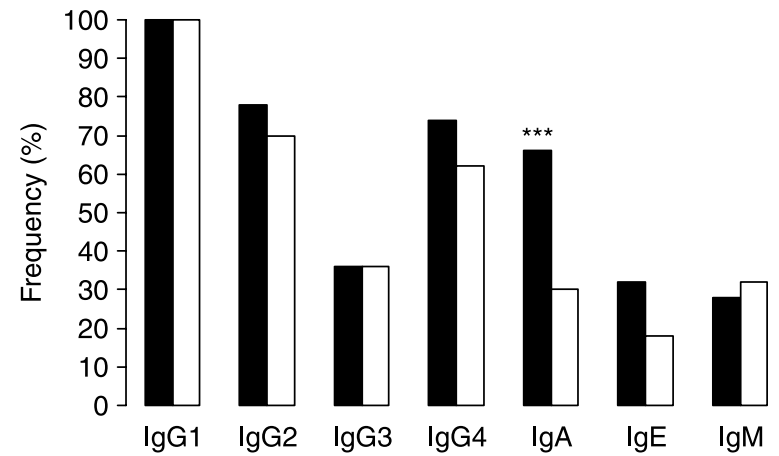

Figure 1 Frequency of different isotype- and subclass-specific IA$2 \mathrm{~A}$ in newly diagnosed type 1 diabetes patients with an IA-2 restricted response ( $\square$ ) and in those with a broad autoantibody response $(\square) .{ }^{\star \star \star} P=0.001\left(\chi^{2}\right.$-statistics).

(data not shown). IA-2 PTP antibodies were the most frequent; $92 \%$ of the restrictive responders and $88 \%$ of the broad responders tested positive for these antibodies $(P=0.74)$ with median levels of 15.3 and $10.7 \mathrm{RU}$ $(P=0.12)$. Cross-reactive IA-2 PTP/IA-2 $\beta$ PTP antibodies were detected in $86 \%$ of the restrictive responders and in $88 \%$ of the broad responders $(P=1.00)$, the median level being 14.4 and 16.5 RU $(P=0.56)$ respectively. IA-2 JM positive responses were observed in 44 and $42 \%(P=1.00)$ of the two groups (median levels 11.6 vs $11.6 \mathrm{RU} ; P=0.92$ ). IA-2 $\beta$ PTP antibodies were infrequent; only $10 \%$ of the restrictive responders and $12 \%$ of the broad responders tested positive for this epitope-specific reactivity $(P=1.00$; median levels 8.4 vs $6.7 \mathrm{RU} ; P=1.00$ ). We also compared the number of positive epitope-specific antibody responses, the median number being two out of four possible $(P=0.80)$ in both the groups.

\section{Isotype-specific antibodies}

All the subjects tested positive for IgG1-IA-2A. Also, IgG2 (78\% of restrictive responders versus $70 \%$ of broad responders) and IgG4 (74\% vs 62\%) subclass antibodies were common, whereas IgG3 (36\% vs $36 \%)$, IgE $(32 \%$ vs $18 \%)$, and $\operatorname{IgM}(28 \%$ vs $32 \%)$ responses were seen less frequently (Fig. 1). The IA-2-restrictive responders tested positive for IgA-IA-2A more often than the broad responders $(66 \%$ vs $30 \%, P=0.001)$. There were also differences in the levels of isotype-specific antibodies (Table 1). IgE-IA-2A levels were higher among the restrictive responders, while IgG1-IA-2A levels were lower in this group. IA-2-restrictive responders had a higher number of positive isotype-specific antibodies $(P=0.04)$, the median being four out of seven in this group, while the corresponding median was three among the broad responders. When all the children were categorized into two groups according to the age at diagnosis using the median age of 9.52 years as the cutoff point, younger subjects had higher IgG3-IA-2A levels than the older subjects among the restrictive responders $(P=0.04)$. This difference was not seen among the broad responders.

\section{IA-2A response in relation to $\mathrm{HLA}$-conferred disease susceptibility}

Children with an IA-2-restrictive response carried more often $(39 / 47 ; 83 \%)$ high and moderate risk HLA genotypes (risk groups 3 and 4 ) than those with a broad autoantibody response $(29 / 48 ; 60 \% ; P=0.03)$. None of the restrictive responders had a protective HLA genotype, whereas two subjects $(4 \%)$ carried this genotype among the broad responders. The strongly predisposing risk haplotype $D R B 1^{*} 0401-D Q B 1^{*} 0302$ was observed to be more frequent among restrictive responders $(37 / 47 ; 79 \%)$ than among broad responders (26/48; 54\%; $P=0.02)$. A combination of any subtype of DR4 with $D Q B 1^{*} 0302$ (the (DR4)-DQB1*0302 haplotype) was also more common among the restrictive responders $(41 / 47 ; 87 \%)$ than the broad responders $(31 / 48 ; 65 \% ; P=0.02)$. The genotype (DR 1/10)-DQB1*0501/DRB1*0401-DQB1*0302 (risk group 3) was seen more frequently among restrictive responders $(13 / 47 ; 28 \%)$ than among broad responders $(2 / 48,4 \% ; P=0.004)$. There was no difference between the groups in terms of the frequency of the high-risk genotype (DR3)-DQA1*05-DQB1*02/DRB1*0401$D Q B 1 * 0302$ (risk group $4 ; 9 / 47$ vs $9 / 48 ; 19 \%$ vs $19 \%$ ).

\section{Family history of T1D}

No difference could be seen in the family history of T1D between the IA-2-restrictive responders and the broad

Table 1 Positive isotype-specific islet antigen 2 antibodies (IA-2A) levels expressed as s.D. scores in IA-2-restrictive and -broad responders among children with newly diagnosed type 1 diabetes. Data are expressed as medians (range).

\begin{tabular}{|c|c|c|c|c|c|}
\hline & $n$ & $\begin{array}{l}\text { Restrictive } \\
\text { responders }\end{array}$ & $n$ & Broad responders & $P$ value \\
\hline $\lg G 1$ & 50 & $1340.8(46.7 ; 3770)$ & 50 & $1761.1(105 ; 3946)$ & 0.049 \\
\hline $\lg \mathrm{g} 2$ & 39 & $45.9(5.2 ; 3227)$ & 35 & $54.5(12.2 ; 638)$ & 0.552 \\
\hline $\operatorname{lgG} 3$ & 18 & $11.0(5.0 ; 211)$ & 18 & $9.8(4.9 ; 134)$ & 0.681 \\
\hline $\operatorname{lgG} 4$ & 37 & $14.2(5.4 ; 187)$ & 31 & $11.8(4.8 ; 59.8)$ & 0.926 \\
\hline $\lg A$ & 33 & $9.3(3.1 ; 52.8)$ & 15 & $9.1(3.4 ; 153)$ & 0.920 \\
\hline $\lg \mathrm{E}$ & 16 & $26.7(3.7 ; 53.4)$ & 9 & $8.6(4.8 ; 105)$ & 0.025 \\
\hline $\lg M$ & 14 & $11.7(4.9 ; 103)$ & 16 & $22.2(5.0 ; 482)$ & 0.430 \\
\hline
\end{tabular}


responders. Six children $(6 / 47 ; 13 \%)$ among the IA-2restrictive responders had at least one family member affected by T1D, whereas the corresponding proportion was $20 \%(10 / 49 ; \quad P=0.47)$ among the broad responders.

\section{Metabolic decompensation at diagnosis}

No significant differences were observed in the markers of the degree of metabolic decompensation at diagnosis between the restrictive and broad responders (median blood glucose concentration $23.2 \mathrm{mmol} / \mathrm{l}$ in the restrictive responders versus $23.6 \mathrm{mmol} / \mathrm{l}$ in the broad responders $(P=0.97)$, the median $\mathrm{pH} 7.38$ vs 7.37 $(P=0.44)$ and the median $\beta$-hydroxybutyrate concentration $1.99 \mathrm{mmol} / \mathrm{l}$ versus $1.66 \mathrm{mmol} / \mathrm{l}(P=0.66))$. The frequency of diabetic ketoacidosis defined as $\mathrm{pH}<$ 7.30 was $23 \%(11 / 48)$ among the restrictive responders and $14 \%(7 / 49 ; P=0.41)$ among the broad responders.

\section{Discussion}

When planning this study, we hypothesized that the humoral immune response against the IA-2/IA- $2 \beta$ protein would be broader among the broad responders than among the IA-2-restrictive responders. Since the humoral immune system was already attacking multiple autoantigens, namely GAD, insulin, and IA-2 in the broad responders, we expected this phenomenon to be reflected by a broad activation also at the level of epitope and isotype specificity to a single antigen. Such a hypothesis is in line with the epitope spreading seen in prediabetes and the isotype switching/spreading reported for both GAD $(19)$ and IA-2 $(9,20)$ in preclinical T1D. To our surprise, the isotype-specific response to IA-2 turned out to be broader among the IA-2-restrictive responders. These observations show that although the humoral immune system of IA-2-restrictive responders is targeting only one of the biochemically characterized $\beta$-cell autoantigens, it is responding to this antigen with a vast array of different immunoglobulin isotypes. Among the broad responders, the titers of IgG1 subclass IA-2A were somewhat higher but the number of various immunoglobulin isotypes targeting IA-2/IA- $2 \beta$ proteins was fewer. The higher levels of IgE class IA-2A and lower IgG1 subclass levels among the restrictive responders point toward an IA-2-specific regulatory or Th2polarized immune response. There was, however, no significant difference in the levels of IgG4 subclass antibodies implicated as reflecting a Th2-biased response or IgG3 subclass antibodies perceived as representing a Th1-polarized response.

There was no difference in the age at diagnosis between those positive for IgE subclass IA-2A and the negative ones. One would have expected IgE-positive children to be older at the time of diagnosis based on our earlier observation that an IgE class IA-2 response protects from progression to T1D among IA-2A-positive children $(17,20)$.

There is a variety of studies in which IA-2 epitopeand isotype-specific antibodies have been analyzed in subjects with preclinical $\mathrm{T} 1 \mathrm{D}$, and their role in predicting disease progression has been assessed. For example, IgG4 subclass IA-2A (21) as well as IgE class IA-2A $(17,20)$ have been associated with protection from progression to T1D. JM-specific IA-2A have been shown to be one of the first epitope-specific IA-2A to appear in young children (9), and they have been associated with a greater risk of progression to overt T1D $(9,17,20)$. There are, however, fewer studies measuring the same parameters among patients with newly diagnosed T1D. Our findings on the frequencies of the different epitope-specific IA-2A are in line with these previous studies, where IA-2A binding specifically to the PTP epitope or cross-reacting with the IA-2/IA-2 $\beta$ PTP epitopes were detected most frequently, while responses targeting the PTP epitope of IA- $2 \beta$ were rare $(4-6,8)$. In studies where isotype-specific IA-2A have been measured in patients with newly diagnosed T1D, IgG1 subclass antibodies have been observed to be the dominant isotype-specific antibody reactivity, although they have not been detected in all the subjects (21-23). Other IgG subclass IA-2A as well as IgE and IgA IA-2A have been observed less frequently, whereas IgM class IA-2A have not been detected (23) or analyzed at all $(21,22)$ in those studies. The present study is in line with previous findings in that IgG1 subclass IA-2A were most prominent (Fig. 1). We in fact observed such antibodies in all of our subjects, possibly due to their high-titer total IA-2 responses. In our series, IgG2 was the second most frequent antibody subclass followed by IgG4. We also detected IgM class IA-2A.

In our series of 100 children with newly diagnosed T1D, those with an IA-2-restrictive humoral immune response carried a stronger HLA-conferred susceptibility to T1D than those with a broad response, but this is due to the specific association with the strongly predisposing $D R B 1 * 0401-D Q B 1^{*} 0302$ haplotype. There was in fact no difference in the frequency of the highest risk associated (DR3)-DQA1*05-DQB1*02/DRB1*0401$D Q B 1^{*} 0302$ genotype. This finding is consistent with previous observations according to which children with (DR4)-DQB1*0302 were shown to test more frequently positive for both IAA and IA-2A and to have higher IAA and IA-2A levels $(11,12)$.

The association between the humoral immune response restricted to the IA-2/IA- $2 \beta$ antigen and a specific HLA risk haplotype implies the possibility of a different pathogenetic mechanism of T1D operating in those children with an IA-2-restricted response. IA-2 might be the primary autoantigen in these children, although one cannot exclude that they may test positive for other diabetes-associated autoantibodies not analyzed in the present study, such as antibodies to the recently characterized zinc transporter ZnT8 (24). 
In general, IA-2A have been shown to be the last appearing antibody reactivity during preclinical diabetes among those with multiple autoantibodies, where their emergence probably reflects antigenic spreading of an ongoing autoimmune response $(25,26)$. In addition to the class II HLA DRB1*0401-DQB1*0302 haplotype, factors controlling the IA-2-restrictive pathogenesis of T1D might include other genetic loci. In previous studies in prediabetic children, the insulin gene INS -(-23)-HphI polymorphism (12), the cytotoxic T-lymphocyte-associated protein 4 (CTLA4) +49 or CT60 polymorphisms (12), or the Arg620Trp variant in the lymphoid tyrosine phosphatase encoding the PTPN22 gene (27) did not affect the production of IA-2A, but the INS and PTPN22 polymorphisms were observed to be associated with the early appearance of IAA and to contribute to the pathogenesis of T1D by initiating/modifying insulinspecific immunity. Further studies are required to unravel the factors influencing the IA-2-restricted response and the possible pathogenetic mechanism behind.

In the present study, we included 50 children testing positive for only IA-2A out of three molecular T1Dassociated autoantibodies at the time of diagnosis and 50 who tested positive for all three molecular antibodies. All children from the Finnish Pediatric Diabetes Register who were eligible for the study design could not be included because the analyses of isotypespecific antibodies are relatively expensive. Since there were no samples available from the time period before the diagnosis of T1D, we cannot rule out the possibility that the children who tested positive for IA-2A only at diagnosis actually had other autoantibodies during the prediabetic period. IAA in particular have been observed to fluctuate between positivity and negativity in children with increased genetic diabetes susceptibility (28). However, transient autoantibody positivity has been reported to be a rare phenomenon especially in children with multiple autoantibodies $(28,29)$. In fact among 1005 children with increased genetic risk for T1D, only one child tested positive for two autoantibodies at some point but later seroconverted to positivity for only one antibody (28). Prospective studies with samples taken before the diagnosis are needed to confirm whether IA-2-restrictive responders truly have a humoral immune response restricted to only that autoantigen.

Positivity for a single autoantibody usually reflects harmless, non-progressive $\beta$-cell autoimmunity, whereas positivity for multiple autoantibodies represents a progressive disease process in subjects at risk for T1D (30). The present observations imply that isolated IA-2A positivity might indicate progressive $\beta$-cell autoimmunity, as it was more common among patients with newly diagnosed T1D than isolated IAA or GADA positivity and was associated with the HLA allele conferring the strongest disease susceptibility. Accordingly, one may consider whether subjects with isolated IA-2A positivity should be included or not in the target group for any immune intervention aimed at the prevention of T1D.

We conclude that there are differences in the humoral immune response to the IA-2 autoantigen between newly diagnosed T1D patients with an IA-2-restrictive antibody profile and those positive for multiple autoantibodies. The restrictive response was characterized by a broader isotype-specific response to the IA-2 antigen and an association with the HLA risk haplotype DRB 1*0401-DQB1*0302 implicating a different pathogenetic mechanism in those with a restricted IA-2specific response.

\section{Acknowledgements}

This study was supported by the Type 1 Diabetes Targeted Program co-funded by the Research Council for Health, Academy of Finland, the Juvenile Diabetes Foundation International and the Sigrid Jusélius Foundation, the Juvenile Diabetes Research Foundation (grant 1-2006-267), the Novo Nordisk Foundation, and the Liv and Hälsa Fund.

\section{References}

1 Knip M. Disease-associated autoimmunity and prevention of insulin-dependent diabetes mellitus. Annals of Medicine 199729 447-451.

2 Ort T, Voronov S, Guo J, Zawalich K, Froehner SC, Zawalich W \& Solimena M. Dephosphorylation of beta2-syntrophin and $\mathrm{Ca} 2+$ /mu-calpain-mediated cleavage of ICA512 upon stimulation of insulin secretion. EMBO Journal 200120 4013-4023.

3 Doi A, Shono T, Nishi M, Furuta H, Sasaki H \& Nanjo K. IA-2beta, but not IA-2, is induced by ghrelin and inhibits glucose-stimulated insulin secretion. PNAS $2006 \mathbf{1 0 3} 885-890$.

4 Lampasona V, Bearzatto M, Genovese S, Bosi E, Ferrari M \& Bonifacio E. Autoantibodies in insulin-dependent diabetes recognize distinct cytoplasmic domains of the protein tyrosine phosphatase-like IA-2 autoantigen. Journal of Immunology 1996 157 2707-2711.

5 Bonifacio E, Lampasona V \& Bingley PJ. IA-2 (islet cell antigen 512 ) is the primary target of humoral autoimmunity against type 1 diabetes-associated tyrosine phosphatase autoantigens. Journal of Immunology $1998 \mathbf{1 6 1} 2648-2654$.

6 Hatfield EC, Hawkes CJ, Payton MA \& Christie MR. Cross reactivity between IA-2 and phogrin/IA-2 beta in binding of autoantibodies in IDDM. Diabetologia 199740 1327-1333.

7 Notkins AL, Zhang B, Matsumoto Y \& Lan MS. Comparison of IA-2 with IA-2beta and with six other members of the protein tyrosine phosphatase family: recognition of antigenic determinants by IDDM sera. Journal of Autoimmunity 199710 245-250.

8 Kawasaki E, Yu L, Rewers MJ, Hutton JC \& Eisenbarth GS. Definition of multiple ICA512/phogrin autoantibody epitopes and detection of intramolecular epitope spreading in relatives of patients with type 1 diabetes. Diabetes $1998 \mathbf{4 7} 733-742$.

9 Naserke HE, Ziegler AG, Lampasona V \& Bonifacio E. Early development and spreading of autoantibodies to epitopes of IA-2 and their association with progression to type 1 diabetes. Journal of Immunology $1998 \mathbf{1 6 1} 6963-6969$.

$10 \mathrm{Kolb}$ H. Benign versus destructive insulitis. Diabetes/Metabolism Reviews 199713 139-146. 
11 Knip M, Kukko M, Kulmala P, Veijola R, Simell O, Åkerblom HK \& Ilonen J. Humoral beta-cell autoimmunity in relation to HLAdefined disease susceptibility in preclinical and clinical type 1 diabetes. American Journal of Medical Genetics 2002115 48-54.

12 Hermann R, Laine AP, Veijola R, Vahlberg T, Simell S, Lähde J, Simell O, Knip M \& Ilonen J. The effect of HLA class II, insulin and CTLA4 gene regions on the development of humoral beta cell autoimmunity. Diabetologia $2005 \mathbf{4 8} 1766-1775$.

13 Savola K, Bonifacio E, Sabbah E, Kulmala P, Vähäsalo P, Karjalainen J, Tuomilehto-Wolf E, Meriläinen J, Åkerblom HK \& Knip M. IA-2 antibodies - a sensitive marker of IDDM with clinical onset in childhood and adolescence. Childhood Diabetes in Finland Study Group. Diabetologia 199841 424-429.

14 Ronkainen MS, Hämäläinen AM, Koskela P, Akerblom HK, Knip M \& Finnish TRIGR Study Group, . Pregnancy induces nonimmunoglobulin insulin-binding activity in both maternal and cord blood serum. Clinical and Experimental Immunology $2001 \mathbf{1 2 4}$ 190-196.

15 Savola K, Sabbah E, Kulmala P, Vähäsalo P, Ilonen J \& Knip M. Autoantibodies associated with type I diabetes mellitus persist after diagnosis in children. Diabetologia 199841 1293-1297.

16 Bottazzo GF, Florin-Christensen A \& Doniach D. Islet-cell antibodies in diabetes mellitus with autoimmune polyendocrine deficiencies. Lancet 1974 2 1279-1283.

17 Hoppu S, Härkönen T, Ronkainen MS, Åkerblom HK, Knip M \& Childhood Diabetes in Finland Study Group. IA-2 antibody epitopes and isotypes during the prediabetic process in siblings of children with type 1 diabetes. Journal of Autoimmunity 200423 361-370.

18 Hermann R, Turpeinen H, Laine AP, Veijola R, Knip M, Simell O, Sipilä I, Åkerblom HK \& Ilonen J. HLA DR-DQ-encoded genetic determinants of childhood-onset type 1 diabetes in Finland: an analysis of 622 nuclear families. Tissue Antigens $2003 \mathbf{6 2}$ 162-169.

19 Ronkainen M, Hoppu S, Korhonen S, Simell S, Veijola R, Ilonen J, Simell O \& Knip M. Early epitope- and isotype-specific humoral immune responses to GAD65 in young children with genetic susceptibility to type 1 diabetes. European Journal of Endocrinology 2006155 633-642.

20 Hoppu S, Härkönen T, Ronkainen MS, Simell S, Hekkala A, Toivonen A, Ilonen J, Simell O \& Knip M. IA-2 antibody isotypes and epitope specificity during the prediabetic process in children with HLA-conferred susceptibility to type I diabetes. Clinical and Experimental Immunology $2006 \mathbf{1 4 4} 59-66$.

21 Seissler J, Eikamp K, Schott M, Scherbaum WA \& DENIS Study Group. IA-2 autoantibodies restricted to the IgG4 subclass are associated with protection from type 1 diabetes. Hormone and Metabolic Research 200234 186-191.
$22 \mathrm{Ng} \mathrm{WY}$, Lui KF, Cheah JS \& Thai AC. IgG1 subclass dominates autoimmune response to tyrosine phosphatase-like molecule IA-2 in Chinese type 1 diabetes patients. Hormone and Metabolic Research $200234596-600$.

23 Hawa MI, Fava D, Medici F, Deng YJ, Notkins AL, De Mattia G \& Leslie RD. Antibodies to IA-2 and GAD65 in type 1 and type 2 diabetes: isotype restriction and polyclonality. Diabetes Care 2000 23 228-233.

24 Wenzlau JM, Juhl K, Yu L, Moua O, Sarkar SA, Gottlieb P, Rewers M, Eisenbarth GS, Jensen J, Davidson HW \& Hutton JC. The cation transporter ZnT8 (Slc30A8) is a major autoantigen in human type 1 diabetes. PNAS $2007 \mathbf{1 0 4} 17040-17045$.

25 Yu L, Rewers M, Gianini R, Kawasaki E, Zhang Y, Verge C, Chase P, Klingensmith G, Erlich H, Norris J \& Eisenbarth GS. Antiislet autoantibodies usually develop sequentially rather than simultaneously. Journal of Clinical Endocrinology and Metabolism $1996 \mathbf{8 1}$ 4264-4667.

26 Kupila A, Keskinen P, Simell T, Erkkilä S, Arvilommi P, Korhonen S, Kimpimäki T, Sjöroos M, Ronkainen M, Ilonen J, Knip M \& Simell O. Genetic risk determines the emergence of diabetes-associated autoantibodies in young children. Diabetes $200251646-651$.

27 Hermann R, Lipponen K, Kiviniemi M, Kakko T, Veijola R, Simell O, Knip M \& Ilonen J. Lymphoid tyrosine phosphatase (LYP/PTPN22) Arg620Trp variant regulates insulin autoimmunity and progression to type 1 diabetes. Diabetologia 200649 1198-1208.

28 Kimpimäki T, Kulmala P, Savola K, Kupila A, Korhonen S, Simell T, Ilonen J, Simell $O$ \& Knip M. Natural history of $\beta$-cell autoimmunity in young children with increased genetic susceptibility to type 1 diabetes recruited from the general population. Journal of Clinical Endocrinology and Metabolism $2002 \mathbf{8 7}$ 4572-4579.

$29 \mathrm{Yu}$ J, Yu L, Bugawan TL, Erlich HA, Barriga K, Hoffman M, Rewers M \& Eisenbarth GS. Transient anti-islet autoantibodies: infrequent occurrence and lack of association with "genetic" risk factors. Journal of Clinical Endocrinology and Metabolism $2000 \mathbf{8 5}$ 2421-2428.

30 Mrena S, Savola K, Kulmala P, Åkerblom HK, Knip M \& the Childhood Diabetes in Finland Study Group. Natural course of preclinical type 1 diabetes in siblings of affected children. Acta Paediatrica 200392 1403-1410.

Received 12 March 2008

Accepted 26 March 2008 


\section{Appendix}

The Finnish Pediatric Diabetes Register and Biobank comprise the following investigators:

Principal Investigator: Mikael Knip (Hospital for Children and Adolescents, Helsinki University Central Hospital),

Steering Committee: Per-Henrik Groop (Folkhälsan Research Institute), Jorma Ilonen (Immunogenetics Laboratory, University of Turku), Jorma Komulainen (Department of Pediatrics, Kuopio University Hospital), Else-Maj Lammi (Hospital for Children and Adolescents, Helsinki University Central Hospital), Raisa Lounamaa (Department of Pediatrics, Central Hospital of Central Finland), Marja-Terttu Saha (Department of Pediatrics, Tampere University Hospital), Olli Simell (Chair, Department of Pediatrics, Turku University Central Hospital), Ilkka Sipilä (Hospital for Children and Adolescents, Helsinki University Central Hospital), Timo Talvitie (Department of Pediatrics, South Ostrobothnia Central Hospital), Outi Vaarala (Department of Viral Disease and Immunology, National Public Health Institute), Riitta Veijola (Department of Pediatrics, Oulu University Hospital),

Locally responsible investigators: Peter Floman (Department of Pediatrics, Porvoo Hospital), Brage Gustafsson (Department of Pediatrics, Jakobstad Hospital), Maarit Haapalehto (Department of Pediatrics, Rauma Hospital), Carita Holm (Department of Pediatrics, Satakunta Central Hospital), Christian Johansson (Department of Pediatrics, Åland Central Hospital), Eero Kaprio (Department of Pediatrics, Peijas Hospital), Tuija Kautto (Department of Pediatrics, Kymenlaakso Central
Hospital), Pirkko Keronen (Department of Pediatrics, Västra Nyland Hospital), Anne Kinnula (Department of Pediatrics, Salo Hospital), Jorma Komulainen (Department of Pediatrics, Kuopio University Hospital), Paavo Korpela (Department of Pediatrics, Kanta-Häme Central Hospital), Pentti Lautala (Department of Pediatrics, Päijät-Häme Central Hospital), Kaija Lindström (Department of Pediatrics, Hyvinkää Hospital), Ulla Niemi (Department of Pediatrics, Lounais-Häme Hospital), Anja Nuuja (Department of Pediatrics, Central Hospital of Central Finland), Päivi Nykänen (Department of Pediatrics, Mikkeli Central Hospital), Seppo Pöntynen (Department of Pediatrics, Central Hospital of Lapland), Anne-Putto-Laurila (Department of Pediatrics, Turku University Central Hospital), Marja-Terttu Saha (Department of Pediatrics, Tampere University Hospital), Juhani Sankila (Department of Pediatrics, Savonlinna Central Hospital), Ilkka Sipilä (Hospital for Children and Adolescents, Helsinki University Central Hospital), Leena Taittonen (Department of Pediatrics, Vaasa Central Hospital), Timo Talvitie (Department of Pediatrics, South Ostrobothnia Central Hospital), Päivi Tapanainen (Department of Pediatrics, Oulu University Hospital), Tapani Uotila (Department of Pediatrics, North Karelia Central Hospital), Marita Uro (Department of Pediatrics, Lohja Hospital), Pentti Varimo (Department of Pediatrics, Kainuu Central Hospital), Gunilla Wetterstrand (Department of Pediatrics, Jorvi Hospital), Ritva Virransalo (Department of Pediatrics, South Karelia Central Hospital), Pentti Vuolukka (Department of Pediatrics, Länsi-Pohja Central Hospital), Olli Vuorinen Department of Pediatrics (Central Ostrobothnia Central Hospital). 